\title{
Correction to: Advancing interdisciplinary research in head and neck cancer through a multicenter longitudinal prospective cohort study: the NETherlands QUality of life and Blomedical Cohort (NET-QUBIC) data warehouse and biobank
}

\author{
I. M. Verdonck-de Leeuw ${ }^{1,2^{*}}$, F. Jansen ${ }^{1,2}$, R. H. Brakenhoff ${ }^{1}$, J. A. Langendijk ${ }^{3}$, R. Takes ${ }^{4}$, C. H. J. Terhaard ${ }^{5}$, \\ R. J. Baatenburg de Jong ${ }^{6}$, J. H. Smit ${ }^{7}$ and C. R. Leemans ${ }^{1}$
}

Correction to: BMC Cancer (2019) 19:765

https://doi.org/10.1186/s12885-019-5866-z

Following publication of the original article [1], the authors reported the name of R.J. Baatenburg de Jong was incorrectly tagged in the HTML version of the article. The correct tagging is:

Given name: R (Robert)

Middle name: $J$

Family name: Baatenburg de Jong

The original article [1] has been corrected.

\begin{abstract}
Author details
'Department of Otolaryngology-Head and Neck Surgery, Cancer Center Amsterdam, Amsterdam UMC, Vrije Universiteit Amsterdam, PO BOX 7057, 1007 MB Amsterdam, The Netherlands. ${ }^{2}$ Department of Clinical, Neuro and Development Psychology, Vrije Universiteit Amsterdam, Amsterdam Public Health Research Institute, Amsterdam, The Netherlands. ${ }^{3}$ Department of Radiation Oncology, University Medical Center Groningen, University of Groningen, Groningen, The Netherlands. ${ }^{4}$ Department of

Otolaryngology-Head and Neck Surgery, Radboud University Medical Center, Nijmegen, The Netherlands. ${ }^{5}$ Department of Radiation Oncology, University Medical Center, Utrecht, The Netherlands. ${ }^{6}$ Department of Otolaryngology and Head and Neck Surgery, Erasmus Cancer Institute, ErasmusMC, Rotterdam, the Netherlands. ${ }^{7}$ Department of Psychiatry, Neuroscience Campus Amsterdam and Amsterdam Public Health Research Institute, Amsterdam UMC, location VU University Medical Center, Amsterdam, The Netherlands.
\end{abstract}

\footnotetext{
*Correspondence: im.verdonck@amsterdamumc.nl; im.verdonck@vumc.nl 'Department of Otolaryngology-Head and Neck Surgery, Cancer Center Amsterdam, Amsterdam UMC, Vrije Universiteit Amsterdam, PO BOX 7057, 1007 MB Amsterdam, The Netherlands

${ }^{2}$ Department of Clinical, Neuro and Development Psychology, Vrije Universiteit Amsterdam, Amsterdam Public Health Research Institute, Amsterdam, The Netherlands

Full list of author information is available at the end of the article
}

Published online: 22 October 2019

\section{Reference}

1. Verdonck-de Leeuw IM, et al. Advancing interdisciplinary research in head and neck cancer through a multicenter longitudinal prospective cohort study: the NETherlands QUality of life and Blomedical Cohort (NET-QUBIC) data warehouse and biobank. BMC Cancer. 2019;19:765. https://doi.org/10. 1186/s12885-019-5866-z

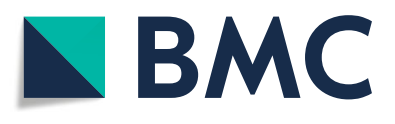

(c) The Author(s). 2019 Open Access This article is distributed under the terms of the Creative Commons Attribution 4.0 International License (http://creativecommons.org/licenses/by/4.0/), which permits unrestricted use, distribution, and reproduction in any medium, provided you give appropriate credit to the original author(s) and the source, provide a link to the Creative Commons license, and indicate if changes were made. The Creative Commons Public Domain Dedication waiver (http://creativecommons.org/publicdomain/zero/1.0/) applies to the data made available in this article, unless otherwise stated. 